\title{
Hologenomic adaptations underlying the evolution of sanguivory in the common vampire bat
}

\author{
M. Lisandra Zepeda Mendoza $\rrbracket^{1,19 \star}$, Zijun Xiong ${ }^{2,3,19}$, Marina Escalera-Zamudio ${ }^{4}{ }^{4}$, Anne Kathrine Runge ${ }^{1}$, \\ Julien Thézé5, Daniel Streicker ${ }^{6}{ }^{6}$, Hannah K. Frank ${ }^{107}$, Elizabeth Loza-Rubio8, Shengmao Liu ${ }^{3}$, \\ Oliver A. Ryder ${ }^{9}{ }^{9}$, Jose Alfredo Samaniego Castruita', Aris Katzourakis ${ }^{5}$, George Pacheco', \\ Blanca Taboada ${ }^{10}$, Ulrike Löber ${ }^{4}$, Oliver G. Pybus ${ }^{5}$, Yang Li ${ }^{3}$, Edith Rojas-Anaya ${ }^{8}$, Kristine Bohmann', \\ Aldo Carmona Baez ${ }^{1,11}$, Carlos F. Arias $\mathbb{1}^{10}$, Shiping Liu ${ }^{3}$, Alex D. Greenwood ${ }^{4,12}$, Mads F. Bertelsen ${ }^{13}$, \\ Nicole E. White ${ }^{14,15}$, Michael Bunce ${ }^{14,15}$, Guojie Zhang ${ }^{2,3,16}$, Thomas Sicheritz-Pontén ${ }^{17 \star}$ \\ and M. P. Thomas Gilbert $\mathbb{B}^{1,15,18 \star}$
}

\begin{abstract}
Adaptation to specialized diets often requires modifications at both genomic and microbiome levels. We applied a hologenomic approach to the common vampire bat (Desmodus rotundus), one of the only three obligate blood-feeding (sanguivorous) mammals, to study the evolution of its complex dietary adaptation. Specifically, we assembled its high-quality reference genome (scaffold $\mathrm{N5O}=26.9 \mathrm{Mb}$, contig $\mathrm{N} 50=36.6 \mathrm{~kb}$ ) and gut metagenome, and compared them against those of insectivorous, frugivorous and carnivorous bats. Our analyses showed a particular common vampire bat genomic landscape regarding integrated viral elements, a dietary and phylogenetic influence on gut microbiome taxonomic and functional profiles, and that both genetic elements harbour key traits related to the nutritional (for example, vitamin and lipid shortage) and non-nutritional (for example, nitrogen waste and osmotic homeostasis) challenges of sanguivory. These findings highlight the value of a holistic study of both the host and its microbiota when attempting to decipher adaptations underlying radical dietary lifestyles.
\end{abstract}

T he order Chiroptera (bats) exhibits a wide variety of dietary specializations, and includes the only three obligate bloodfeeding mammalian species, the vampire bats (family Phyllostomidae, subfamily Desmodontinae). Blood is a challenging dietary source because it consists of an $\sim 78 \%$ liquid phase and a dry-matter phase consisting of $\sim 93 \%$ proteins and only $\sim 1 \%$ carbohydrates $^{1}$, providing very low levels of vitamins ${ }^{2}$, and potentially containing blood-borne pathogens. Vampire bats have evolved numerous key physiological adaptations to this lifestyle, for which the associated genomic changes have not yet been fully characterized due to the lack of an available reference genome. These adaptations include morphological specializations (such as claw-thumbed wings and craniofacial changes including sharp incisors and canines), infrared sensing capacity ${ }^{3}$ for the identification of easily accessible blood vessels in prey ${ }^{4}$, and renal adaptations to the high protein content in its $\operatorname{diet}^{5}$ (such as a high glomerular filtration rate and effective urea excretion). Furthermore, given the high risk of exposure to blood-borne pathogens, another important trait in the vampire bat is its immune system ${ }^{6}$.

Besides genomic adaptations, host-associated microbiota may play an additional, possibly equally important, role in the evolution of vertebrate dietary specialization ${ }^{7}$. Although the functional role of the vampire bat gut microbiome has not been studied, analyses of obligate invertebrate sanguivorous organisms ${ }^{8}$ have shown that the gut microbiota contributes to blood meal digestion ${ }^{9}$, provision of nutrients absent from blood ${ }^{10}$ and to immunological protection ${ }^{11}$. Studies on mammals have shown that the gut microbiome is a key aspect of an organism's digestive capacities (energy harvest, nutrient acquisition and intestinal homeostasis) ${ }^{12}$, and that it also affects phenotypes related to the immune and neuroendocrine systems ${ }^{13,14}$. Furthermore, changes in the gut microbiome are associated with diseases such as diabetes, obesity, irritable bowel syndrome and

\footnotetext{
${ }^{1}$ Centre for GeoGenetics, Natural History Museum of Denmark, University of Copenhagen, Copenhagen, Denmark. ${ }^{2}$ State Key Laboratory of Genetic Resources and Evolution, Kunming Institute of Zoology, Chinese Academy of Sciences, Kunming, China. ${ }^{3}$ China National GeneBank, BGI-Shenzhen, Shenzhen, China. ${ }^{4}$ Department of Wildlife Diseases, Leibniz Institute for Zoo and Wildlife Research (IZW), Berlin, Germany. ${ }^{5}$ Department of Zoology, University of Oxford, Oxford, UK. ${ }^{6}$ Institute of Biodiversity, Animal Health and Comparative Medicine \& MRC-University of Glasgow Centre for Virus Research, University of Glasgow, Glasgow, UK. ' Department of Biology, Stanford University, Stanford, CA, USA. ${ }^{8}$ Centro Nacional de Investigación Disciplinaria en Microbiología Animal-INIFAP, Ciudad de México, Mexico. ${ }^{9}$ San Diego Zoo Institute for Conservation Research, Escondido, CA, USA. ${ }^{10}$ Departamento de Genética del Desarrollo y Fisiología Molecular, Instituto de Biotecnología, Universidad Nacional Autónoma de México, Cuernavaca, Mexico. "Undergraduate Program for Genomic Sciences, Center for Genomic Sciences, National Autonomous University of Mexico, Cuernavaca, Mexico. ${ }^{12}$ Department of Veterinary Medicine, Freie Universität Berlin, Berlin, Germany. ${ }^{13}$ Center for Zoo and Wild Animal Health, Copenhagen Zoo, Frederiksberg, Denmark. ${ }^{14}$ Australian Wildlife Forensic Services, Department of Environment and Agriculture, Curtin University, Perth, Australia. ${ }^{15}$ Trace and Environmental DNA Laboratory, Department of Environment and Agriculture, Curtin University, Perth, Australia. ${ }^{16}$ Centre for Social Evolution, Department of Biology, University of Copenhagen, Copenhagen, Denmark. ${ }^{17}$ Center for Biological Sequence Analysis, Department of Bio and Health Informatics, Technical University of Denmark, Kongens Lyngby, Denmark. ${ }^{18}$ Norwegian University of Science and Technology, University Museum, Trondheim, Norway. ${ }^{19}$ These authors contributed equally: M. Lisandra Zepeda Mendoza and Zijun Xiong. *e-mail: lisandracady@gmail.com; thomas@cbs.dtu.dk; tgilbert@snm.ku.dk
} 
inflammatory bowel disease ${ }^{15-17}$. In response to the growing awareness of the key roles that host-microbiome relationships can play across the spectra of life, various studies have advocated for the 'hologenome' concept ${ }^{18-20}$. In brief, this argues that natural selection acts on both the host and its microbiome (together forming the holobiont); thus, evolutionary studies should incorporate both. The extreme dietary adaptation of vampire bats provides a suitable model to investigate the effect of selection across the genome and microbiome, and thus allows exploration of the role of host-associated microbiome in the evolution of specialized diets.

\section{Results and discussion}

Here, we explore the contributions of both the common vampire bat's nuclear genome and gut microbiome to its adaptation to obligate sanguivory. To this end, we generated both the common vampire bat genome and fecal metagenomic data sets as a proxy to study its gut microbiome, as well as faecal metagenomic data sets from other non-sanguivorous bat species. We used these data sets for comparative genomic and metagenomic analyses. Specifically, we analysed the common vampire bat genomic landscape, the ratio of substitution rates at non-synonymous and synonymous sites $(\mathrm{dN} / \mathrm{d} S)$, putative gene loss and gene family expansion/contraction, and computational predictions of the functional impact of aminoacid substitutions. We also performed microbial taxonomic and functional profiling, identified the microbial taxonomic and functional core of the common vampire bat, and identified enriched microbial taxa and functions. Following a hologenomic approach, we identified elements in both the host genome and microbiome that could have played relevant roles in adaptation to sanguivory.

Genomic landscape. We sequenced and de novo assembled the $\sim 2$ gigabase $(\mathrm{Gb})$ common vampire bat genome using Illumina sequencing technology (Supplementary Information 1). The genome is smaller than that of other mammals, but similar to previously reported bat genomes ${ }^{21}$. The initial assembly $(\sim 100 \times$ mean coverage, scaffold $\mathrm{N} 50=5.5 \mathrm{Mb}$ and $\mathrm{N} 90=933 \mathrm{~kb}$; Supplementary Figs. 1 and 2) was subsequently improved using the in vitro proximity ligation-based technology for assembly contiguity refinement developed by Dovetail Genomics ${ }^{22}$. We obtained a final highquality assembly with scaffold $\mathrm{N} 50=26.9 \mathrm{Mb}$ and $\mathrm{N} 90=9.46 \mathrm{Mb}$, contig $\mathrm{N} 50=36.6 \mathrm{~kb}$ and $\mathrm{N} 90=8.8 \mathrm{~kb}$ (Supplementary Table 1, Supplementary Fig. 3 and Supplementary Information 1). We used our annotated common vampire bat genome (see MethodsProtein-coding gene and functional annotation) for comparative genomic analyses with publicly available bat genomes and other mammalian genomes (Supplementary Table 2 and Fig. 1).

Repetitive elements can significantly contribute to genome evolution. Thus, for a genomic landscape characterization, we first compared transposable elements in the common vampire bat genome to those within the genomes of non-phyllostomid bats with other diets: the carnivorous Megaderma lyra (greater false vampire bat, Megadermatidae), the insectivorous Pteronotus parnellii (Parnell's moustached bat, Mormoopidae) and the frugivorous Pteropus vampyrus (large flying fox, Pteropodidae) (Fig. 1, Supplementary Table 3 and Supplementary Note 1). We identified a 1.6- to 2.26-fold higher copy number of the MULE-MuDR transposon in the common vampire bat genome relative to the other bat genomes. The high mutagenic capacity of MULE-MuDR has been demonstrated to have played a critical role in the evolution of some plants ${ }^{23}$. Furthermore, transposable elements in general may cause structural or functional changes within the genome and alter epigenetic regulation of the genes into which they are inserted ${ }^{24}$. Therefore, we explored whether these elements might have also played a role in the evolution of sanguivory by analysing their genomic location in the nuclear genome of the common vampire bat. We found that the identified common vampire bat transposable elements, MULE-MuDR elements in particular, were

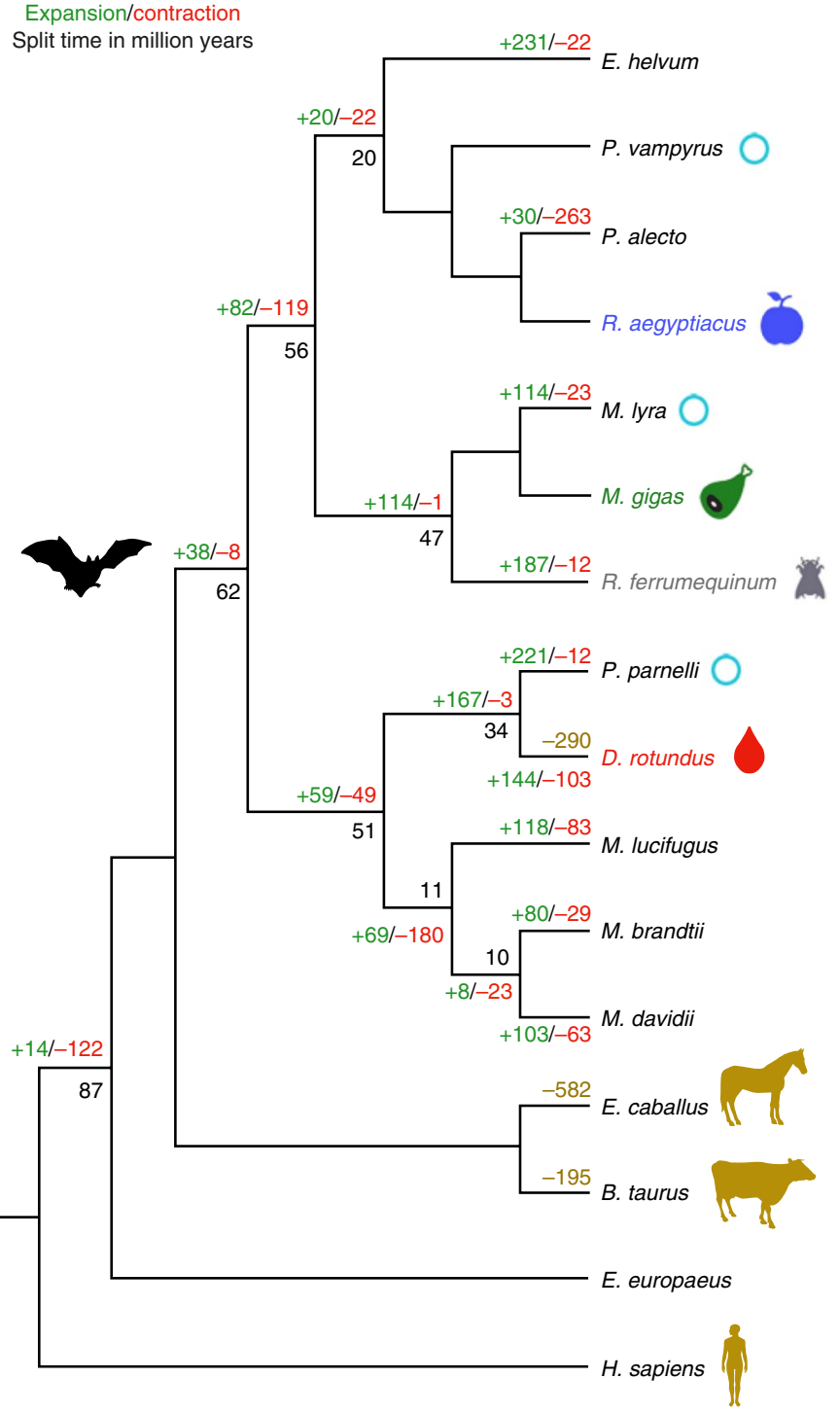

Fig. 1 | Comparative genomic analyses. Species with a light blue circle were used for comparison of transposable elements. In golden colour are the silhouettes of the species used for the identification of putative lost genes and their corresponding number of putatively lost genes compared to the human genome. The number of gene families under expansion (green) and contraction (red) in Chiroptera using E. europaeus as an outgroup are indicated. Estimated dates of nodes (in million years) are indicated in black. The gut microbiomes of bats with different diets derive from $R$. aegyptiacus (blue, frugivorous), M. gigas (green, carnivorous), R. ferrumequinum (grey, insectivorous) and the common vampire bat (red, sanguivorous).

H. sapiens, Homo sapiens; E. helvum, Eidolon helvum.

located within genomic regions enriched for gene ontology (GO) functions related to the challenges of sanguivory, such as antigen processing and presentation, defence response to viruses, lipid metabolism, and vitamin metabolism (Supplementary Information 2).

A sanguivorous diet facilitates exposure to blood-borne viruses that could lead to an increase in genomic invasion by retroviral and non-retroviral endogenous viral elements (EVEs). Thus, we next characterized their presence in the common vampire bat genome. Compared to previously published EVE studies on nonChiropteran mammals, the common vampire bat exhibits a greater diversity of non-retroviral EVEs in terms of the number of integrations, including endogenized viral genes from avian Bornaviridae 
a

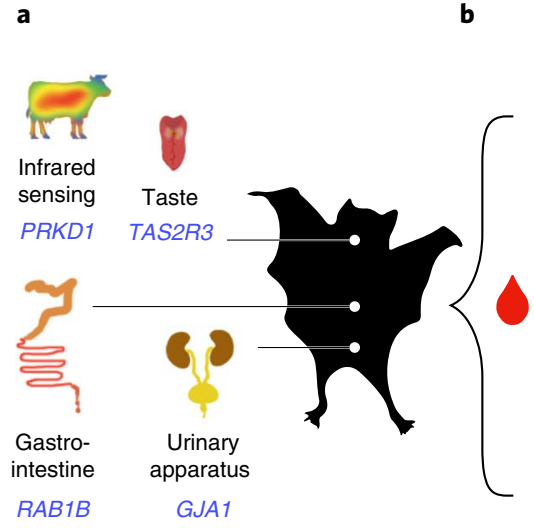$$
\text { RAB }
$$

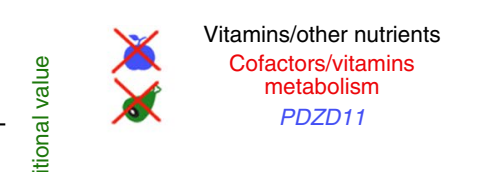
Vitamins/other nutrients Cofactors/vitamins PDZD11

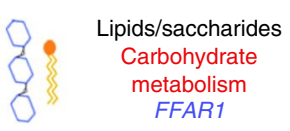

FFAR1

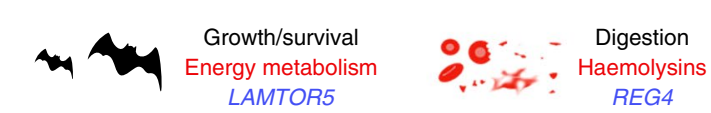

$4 \begin{gathered}\text { Energy metabolism } \\ \text { LAMTOR5 }\end{gathered}$

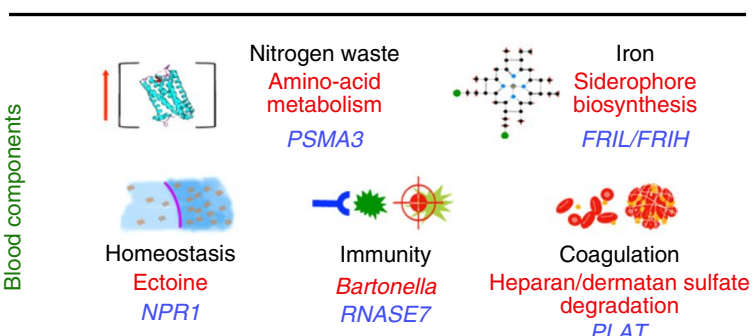

PLAT

Fig. 2 | Dietary challenges overcome by the common vampire bat. a, Adaptational contributions to sanguivory accountable to genomic changes alone (blue labels). b, Adaptational contributions to sanguivory within a hologenomic context (blue labels for host genes; red labels for gut microbial traits).

and Parvoviridae/Dependovirus. However, these findings are not restricted to vampire bats and are similar to those in other bat species $^{25}$ (Supplementary Note 2, Supplementary Fig. 4 and Supplementary Information 3). Surprisingly, and in contrast to the prior expectations given its sanguivorous diet, the diversity of endogenous retroviral elements (ERVs) in the common vampire bat is very low compared to other bat species ${ }^{26}$. The only proviral elements detected were DrERV ${ }^{27}$ and DrgERV, both present in low copy numbers (Supplementary Note 3, Supplementary Fig. 5 and Supplementary Information 4). We hypothesize that genome colonization by ERVs could have been restricted by the genomic adaptations in the common vampire bat genome against ERV insertion and proliferation. In support of our hypothesis, we identified expansion of the anti-retroviral gene TRIM5 family (Viterbi $P=0.00088$, Supplementary Information 5 and Supplementary Note 4).

Genomic adaptations to sanguivory. Feeding specialization often requires morphological and physiological adaptations in traits such as the sensory apparatus (for example, infrared sensing), locomotion, digestion, kidney function and immunity (Fig. 2a and Supplementary Information 6). For example, it has been shown that vampire bats have a loss of sweet taste genes and reduction of bitter taste genes ${ }^{28}$. In agreement, such genes were also identified in our putative gene loss analysis (Supplementary Information 7 and Supplementary Note 5). It is likely that the function of those genes is related to sanguivory, because sweet and bitter taste receptor genes influence glucose homeostasis in humans ${ }^{29}$. Interestingly, we found that the common vampire bat bitter taste receptor gene TAS2R3 has experienced episodic positive selection and shows two speciesspecific positively selected sites (PSSs) on topological domains, one of them having a potential impact on protein function (PROVEAN score $=-4.4$ ) (Supplementary Table 4 and Supplementary Fig. 6). Among the enriched GOs of the differentially evolving genes, we identified functions related to the regulation of RNA splicing, which could be relevant to sanguivory given that $D$. rotundus produces submandibular tissue-specific splicing isoforms to counteract the prey's response to injury ${ }^{30}$ (Supplementary Information 8 and Supplementary Note 6). Regarding the recruitment of alternatively spliced forms, the ganglion-specific splicing of TRPV1 has been found to underlie the vampire bat's infrared sensing ability $^{31}$. Interestingly, we found that $P R K D 1$, which directly modulates the product of TRPV1 $1^{32}$, is positively selected and exhibits species-specific PSSs (branch-site test $P=3.39 \times 10^{-10}$, branch test
$P=1.39 \times 10^{-6}$, Supplementary Information 8). These examples suggest an important role of alternative splicing as a form of regulatory evolution fundamental to sanguivory (Supplementary Note 7). However, it is clear that despite the number of detected genomic features related to sanguivory adaptation, they alone cannot address all of the challenges posed by this diet (Fig. 2b).

Gut microbiome diet and phylogenetic influence. We generated Illumina shotgun metagenome data in order to compare the gut microbiomes of 13 faecal samples from common vampire bats with those of non-sanguivorous non-phyllostomid bats; specifically, eight frugivorous Rousettus aegyptiacus (Egyptian fruit bat, Pteropodidae), five insectivorous Rhinolophus ferrumequinum (greater horseshoe bat, Rhinolophidae) and five carnivorous Macroderma gigas (ghost bat, Megadermatidae) bats (Supplementary Information 9). We obtained a median of $15.8 \mathrm{~Gb}$ of sequencing data ( $\sim 37.6$ million 100 -bp paired-end reads) for each dietary category. After filtering low-quality bases, adaptor sequences and bat-genome-derived reads, we obtained a median of $2.77 \mathrm{~Gb}$ of high-quality data for each species, totalling $86.73 \mathrm{~Gb}$ of data. We identified taxa and functions present only in the common vampire bat microbiome (gut microbiome core), as well as taxa and functions that exhibit statistically significant differences in abundance or contribution to variation between the different microbiomes (Supplementary Information 6 and 8, Supplementary Tables 5 and 6 and Supplementary Note 8).

It has been observed previously that similarity in the taxonomic composition of vertebrate gut microbiomes (including bats) can be influenced by the diet and the phylogenetic relationships of the respective host species ${ }^{33}$. Overall, the common vampire bat microbiome taxonomic composition is more similar to that of the insectivorous and carnivorous bats than to that of the frugivorous bat. This may reflect a phylogenetic influence on the microbiome taxonomic profile (Fig. 3a, Supplementary Fig. 7 and Supplementary Notes 9 and 10). In contrast, the vampire bat microbiome is strikingly different to that of the compared bats at the functional level, which was characterized by the KEGG annotations of the microbial non-redundant gene catalogues assembled from the metagenomic data sets. While there is little differentiation between the functional gut microbiomes of carnivorous, insectivores and frugivorous bats, the common vampire bat functional gut microbiome is almost completely distinct, and exhibits the least intra-species variation between the samples (Fig. 3b, Supplementary Fig. 8 and Supplementary Table 7). 

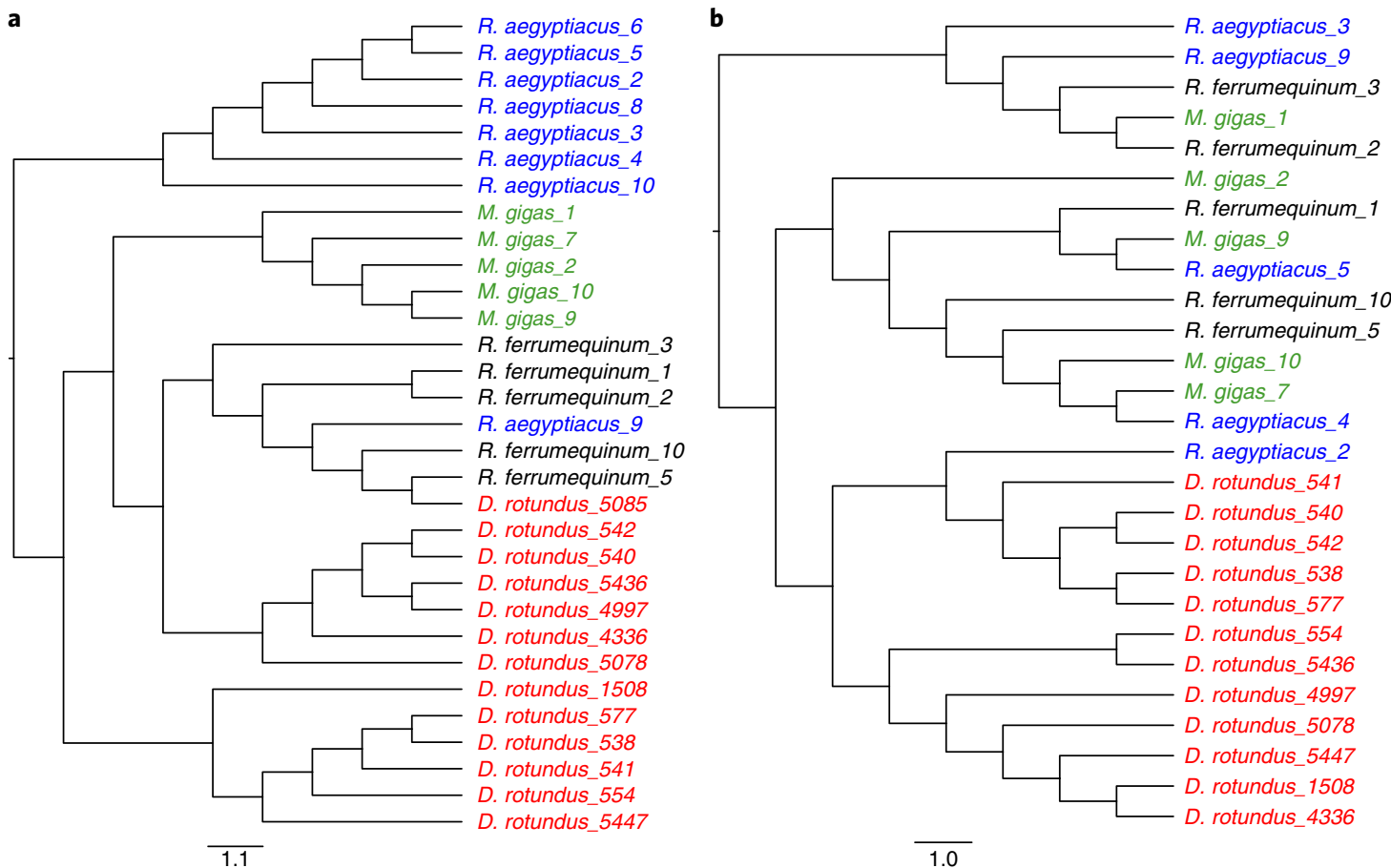

Fig. 3 | Comparison of the taxonomic and functional gut microbiome profiles. D. rotundus (sanguivorous, red), R. ferrumequinum (insectivorous, black), M. gigas (carnivorous, green) and R. aegyptiacus (frugivorous, blue). a, Euclidean distance dendrogram of the microbial presence/absence identifications at the species taxonomical level. $\mathbf{b}$, Euclidean distance dendrogram from the UniProt-identified abundance functions from the normalized samples.

This suggests that the functional profile is less influenced by phylogeny than the taxonomic profile, and that the common vampire bat gut microbiome harbours a set of functions specialized to its extreme diet (Supplementary Note 11). Subsequently, we analysed the comparative genomic and metagenomic results in a hologenomic framework to demonstrate how both components contribute to adaptation to sanguivory (Fig. 4, Supplementary Figs. 9-12, Supplementary Tables 7-9 and Supplementary Information 8 and 10).

The hologenomic framework of sanguivory. Viscosity and subsequent coagulation represent a challenge for ingestion and digestion of blood. Besides developing potent anticoagulants in its saliva $^{34}$, the common vampire bat hologenome addresses this challenge in various ways. For instance, REG4, involved in metaplastic responses of the gastrointestinal epithelium, was found to be under ongoing positive selection (M8a/M8 test $P=0.047$ ) with possible functional impact on its carbohydrate-binding capacity, including binding of the anticoagulant heparin (Supplementary Table 4 and Supplementary Information 8). Furthermore, we identified genes in the common vampire bat microbial functional core from pathways for degradation of heparan sulfate and dermatan sulfate, both being polysaccharides involved in blood coagulation (Supplementary Information 10). We also identified an enrichment in the common vampire bat microbial gene L-asparaginase (Fisher's $P=0.00027$ ), which decreases protein synthesis of coagulation factors $^{35}$ (Supplementary Information 11).

Besides specialized digestion, sanguivory poses other challenges related to the poor nutritional value of blood itself, as well as to the side effects that may arise due to the blood components being the sole dietary source (Fig. 2b). We identified elements in both the genome and gut microbiome that might be involved in solving each of these challenges discussed next.

Hologenomic solutions to nutritional challenges. Low nutrient availability. Obligate sanguivory requires adaptation to very low levels of some nutrients, such as essential amino acids and the vitamin B complex ${ }^{36,37}$, and very high levels of others, such as salt ${ }^{38}$. Our data clearly demonstrate how both the host and its associated gut microbiome have dealt with these challenges. We found the gene LAMTOR5 to be positively selected in the common vampire bat genome (false discovery rate $(F D R)=2.02 \times 10^{-7}$, Supplementary Information 8). This gene is involved in the response to nutrient starvation $^{39}$, suggesting that the common vampire bat metabolism has adapted to the low nutrient content available in blood. Similarly, we identified KOs in the common vampire bat microbial core related to energy and carbohydrate metabolism (Fig. $4 \mathrm{~b}$ and Supplementary Information 11). For example, when compared to the other bats, we identified an enrichment in the common vampire bat microbial genes involved in response to low nutrient availability (RelA/SpoT family protein, Fisher's $P=0.0064$, and guanosine pentaphosphate, Fisher's $P=0.0018$ ), and enzymes in the common vampire bat core involved in the reverse Krebs cycle (Supplementary Information 10 ), which is used by some bacteria to produce carbon compounds from $\mathrm{CO}_{2}$ and water (abundant blood components). We speculate that the presence of such metabolic pathways indicates the growth of specific microbes in the gut's environmental conditions resulting from blood consumption.

The holobiont has also provided solutions to the lack of important nutrients in blood (Fig. 4c and Supplementary Information 8). For example, the PDZD11 gene in the common vampire bat genome, involved in vitamin $B_{5}$ metabolism, evolved faster in the common vampire bat genome relative to the other examined bats (branch test $P=1.97 \times 10^{-10}$ ). We further postulate that the microbiome also contributes in tackling the low nutritional challenge by providing necessary nutrients. For example, compared to the other bat microbiomes, the common vampire bat gut microbiome had the highest number of enriched enzymes related to the biosynthesis of cofactors and vitamins, such as carotenoid (Supplementary Fig. 8B and Supplementary Information 10 and 11). Furthermore, we identified enzymes involved in the metabolism of butyrate, an 


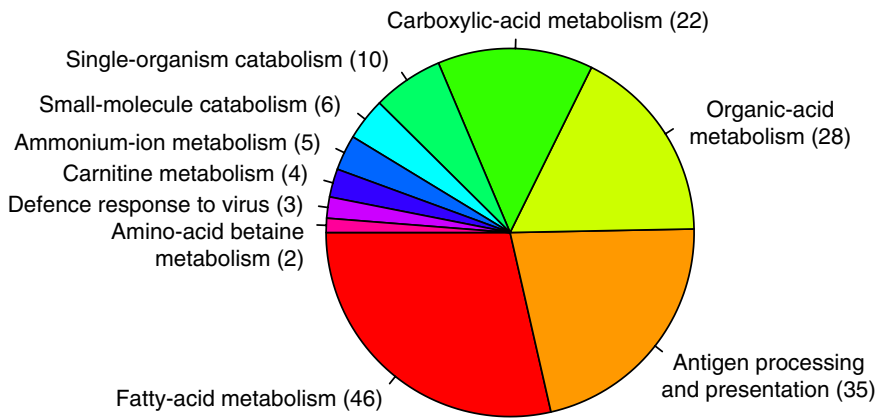

b

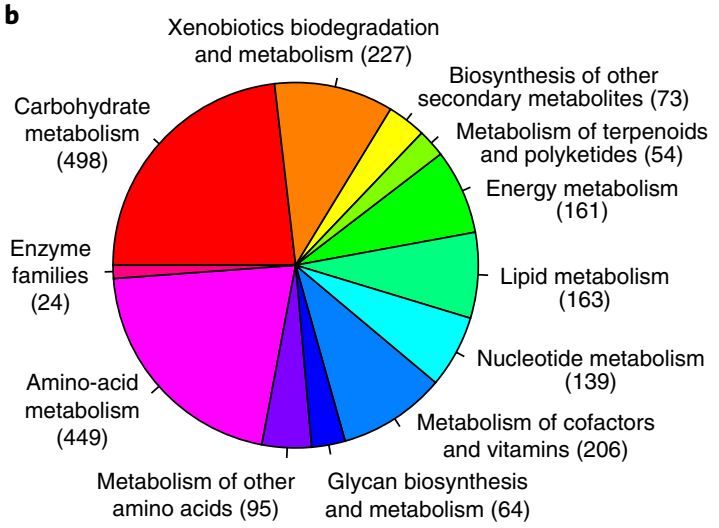

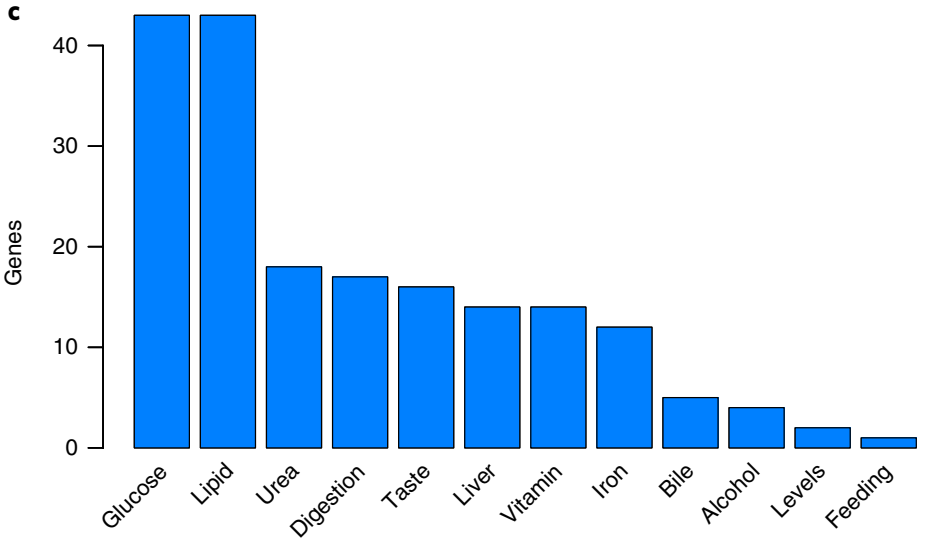

d

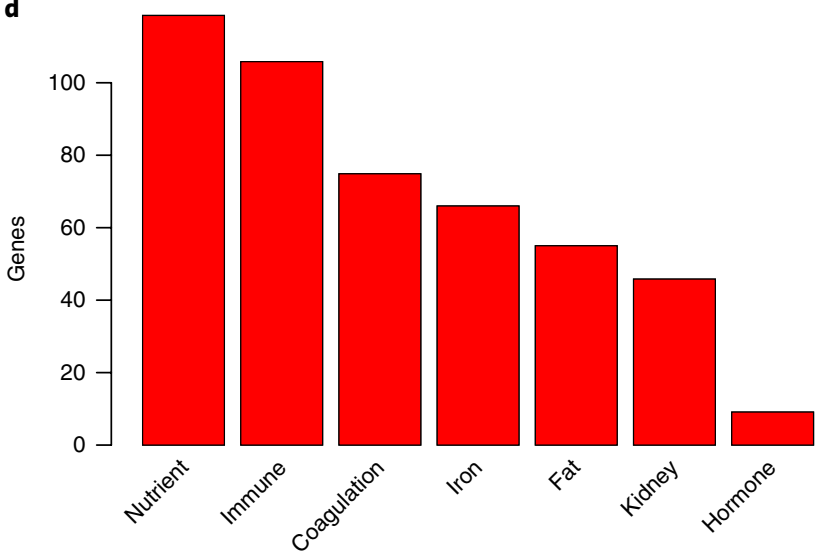

Fig. 4 | Traits in both the genome and gut microbiome with direct roles in the adaptation for sanguivory. a, GOs of the single-copy orthologous genes with a $\mathrm{dN} / \mathrm{d} S$ ratio that is statistically higher or lower in the common vampire bat in comparison to the other bats. $\mathbf{b}$, KOs from the common vampire bat gut microbiome functional core. $\mathbf{c}$, Genes with a $\mathrm{d} N / \mathrm{d} S$ ratio that is statistically higher or lower in the common vampire bat in comparison to the other bats and that are directly associated with sanguivory challenges. d, Taxa and genes from the common vampire bat gut microbiome core directly associated with the adaptation to sanguivory.

important nutrient for cells lining the mammalian colon derived from bacterial fermentation ${ }^{40}$, enriched in the common vampire bat gut microbiome as well as in the vampire bat gut microbiome core (Supplementary Information 10 and 11).

Lipid and glucose assimilation. Besides vitamins and other nutrients, lipids and glucose may not be readily available in blood. Furthermore, vampire bats have a reduced capacity to store energy reserves. In agreement with this, we identified GO enrichment of lipid metabolism on genes with $\mathrm{dN} / \mathrm{dS}$ values statistically higher or lower compared to other bats (Fig. 4a,c and Supplementary Information 8). For example, we identified the gene FFAR1, which plays an important role in glucose homeostasis, as evolving faster in the common vampire bat compared to the other bats (branch test $P=3.68 \times 10^{-5}$ ) and containing amino-acid substitutions with a possible functional impact on its binding ability (Supplementary Table 4). This may enable the common vampire bat to better utilize the available glucose. The common vampire gut microbiome also exhibits unique solutions to the challenge (Fig. 4b,d and Supplementary Information 10 and 11). Differences in the carbohydrate and glycan metabolism functional profile were identified in the principal component analysis (PCA) comparing the different microbiomes (Supplementary Fig. 11), which place the vampire bat gut microbiome profile within a cluster separate from those of the non-sanguivorous bats. Importantly, we identified enrichment in the microbial gene glycerol kinase in the common vampire bat (Fisher's $P=0.0027$ ), which plays a key role in the formation of triacylglycerol and in fat storage, and its deficiency causes symptoms such as hypoglycaemia and lethargy in a mouse model ${ }^{41}$.

Hologenomic solutions to non-nutritional challenges. Immunity. Due to its sanguivorous lifestyle, the common vampire bat risks direct contact with blood-borne pathogens from prey. Consequently, we observed $>280$ bacterial species known to be pathogenic to some mammalian species present exclusively in the common vampire bat gut microbiome (Supplementary Information 12). For example, we identified enrichment of genes from Borrelia (Supplementary Information 11) and Bartonella as one of the most abundant genera in the common vampire bat compared to the other bats (Supplementary Information 13). These bacteria are known to be transmitted by sanguivorous invertebrates (ticks, fleas, mosquitoes and lice). This suggests that the abundance of this genus could be a shared pattern of sanguivorous species. While several studies have elucidated part of the expected genomic immunity-related adaptations $^{6}$, analysis of the full genome enabled us to identify more elements related to immunity, such as defence response to virus and antigen processing and presentation (Fig. 4a,c and Supplementary Information 8). For example, we identified the antimicrobial gene $R N A S E 7$ to be positively selected (branch-site test $P=0.004$ ) and containing amino-acid substitutions that may increase its bactericidal capacity (Supplementary Table 4). In addition, when compared to the gut microbiomes of non-sanguivorous bats, that of the common vampire bat contains a large abundance of potentially protective bacteria, such as Amycolatopsis mediterranei $(P<0.05)$, 
which has been shown to produce antiviral compounds against bacteriophages and poxviruses ${ }^{42}$ (Supplementary Information 13).

Iron assimilation. Iron concentration represents a significant challenge to sanguivory. Although the concentration of free iron ion is not high in the blood, severe haemolysis (for example, during digestion of blood) could result in high levels of iron that, if absorbed in excess, could accumulate and disrupt the normal function in organs such as the liver, heart and pancreas. Interestingly, we identified the light and heavy chains of the iron-storing protein ferritin (encoded by FTL, FTH1) under gene family expansion in the common vampire bat genome (Viterbi $P=0$ and Viterbi $P=0.0012$, respectively, Supplementary Information 5). In addition, we identified an enrichment of the iron-storing protein ferritin (Fisher's $P=0.0014$ ), suggesting that the gut microbiome also contributes to solving this challenge (Fig. 4d and Supplementary Information 11).

Nitrogen waste and blood/osmotic pressure. The high abundance of protein in the blood and its rapid ingestion could lead to accumulation of nitrogenous waste products, primarily urea, which could lead to renal disease-like symptoms (for example, high blood pressure and fluid retention). This challenge is exacerbated by the abundance of salts in blood, which pose additional osmotic and blood pressure challenges. We see at the genome level that this is addressed by a higher rate of evolution in the common vampire bat genes compared to the other bats involved in disposal of excess nitrogen (Fig. 4a,c and Supplementary Information 8), such as PSMA3 (branch test $P=2.08 \times 10^{-7}$ ). This challenge seems to also be addressed by the gut microbiome. The PCA of the copy number of genes involved in amino-acid metabolism distinguishes the common vampire bat in a single cluster separated from the other bat species analysed (Supplementary Fig. 11), suggesting a specialized microbial amino-acid metabolism capacity. We also identified enrichment in the common vampire bat microbial gene urease subunit alpha (ureA, Fisher's $P=0.016$ ) involved in urea degradation (Supplementary Information 11).

\section{Conclusions}

It is clear from our results that the common vampire bat has adapted to sanguivory through a close relationship between its genome and gut microbiome. We identified a phylogenetic and dietary impact on the common vampire bat gut microbiome and uncovered an unexpected genomic viral and repetitive element genomic makeup. We showed that extreme dietary specializations, such as that of the common vampire bat, provide a comparative framework with which to tease apart the relative roles of genomes and microbiomes in adaptation. In conclusion, our study illustrates the benefits of studying the evolution of complex adaptations under a holobiome framework, and suggests that vertebrate adaptation studies that do not account for the action of the hologenome may fail to recover the full complexity of adaptation.

\section{Methods}

Genome sequencing and raw-read processing. We shotgun-sequenced the $D$. rotundus genome using a wing biopsy from a sample collected by the NIH through the Catoctin Wildlife and Zoo in Thurmont, Maryland, USA. The capturing method and dead preservation procedure of the specimen are unknown. The age and sex of the dead individual are unknown. Sampling permits were given to BGI for the sequencing of the specimen, originally as part of the BGI $10 \mathrm{~K}$ genome project. Genomic DNA was extracted at the Laboratory of Genomic Diversity and was fragmented to 2-10 kilobases $(\mathrm{kb})$. Sequencing libraries were constructed with insert sizes $170 \mathrm{bp}-10 \mathrm{~kb}$, according to the Illumina protocol for sequencing on Illumina HiSeq2000 following the manufacturer's instructions. We sequenced reads of $49 \mathrm{bp}$ for the long-insert-size libraries $(2 \mathrm{~kb}, 5 \mathrm{~kb}$ and $10 \mathrm{~kb})$ and $100 \mathrm{bp}$ for the short-insert-size libraries ( $170 \mathrm{bp}, 500 \mathrm{bp}$ and $800 \mathrm{bp}$ ). Sequencing errors were corrected on the basis of the frequency of nucleotide strings of a given length and low-quality reads were filtered out using SOAPfilter ${ }^{43}$ as follows. (1) Remove reads with $>10 \%$ uncalled nucleotides (Ns). (2) For short-insert-size libraries $(<2 \mathrm{~kb})$, reads were removed if the quality score of $>60 \%$ bases was $<7$. For large-insert-size libraries ( $\geq 2 \mathrm{~kb}$ ), reads were removed if the quality score of $>80 \%$ bases was $<7$. (3) Adapter sequences, and duplicate or identical reads were removed. (4) Read pairs were removed if Read 1 and Read 2 were completely identical. (5) For short-insertsize paired-end sequences, reads with overlapping length $\geq 10 \mathrm{bp}$ between the Read 1 and Read2 were removed.

Genome assembly. We estimated the genome size of $D$. rotundus using Kmerfreq by dividing the total number of seven decamers by the peak of the seven decamer Poisson distribution. High-quality reads were assembled using SOAPdenovo ${ }^{43}$ as follows. (1) Short-insert library reads were assembled as initial contigs ignoring the sequence pair information. (2) Reads were aligned to the previously generated contig sequences. Scaffolds were constructed from short-insert-size libraries to large-insert-size libraries by weighting the paired-end relationships between pairs of contigs, with at least three read pairs required forming a connection between any two contigs. (3) Gaps in the scaffolds were closed using GapCloser ${ }^{43}$. Genome quality assessment was performed by downloading a publicly available $D$. rotundus transcriptome ${ }^{45}$ and aligning the transcripts to the genome using BLAT ${ }^{46}$.

Genome contiguity improvement. We prepared two Chicago libraries ${ }^{22}$ using $5 \mu \mathrm{g}$ of high-molecular-weight DNA obtained from $D$. rotundus cultured cells from the San Diego Zoo collection, which were originally derived from a skin sample taken from between the shoulder blades of a D. rotundus individual. Permits for this were obtained from the San Diego Zoo Global. The capturing method and dead preservation procedure of the specimen are unknown. The age and sex of the dead individual are unknown. DNA was extracted with Qiagen Blood and Cell Midi kits according to the manufacturer's instructions. The steps required for building the Chicago libraries were performed as described in ref. ${ }^{22}$. The libraries were sequenced using Illumina HiSeq $25002 \times 100$ bp rapid run. Our initial $D$. rotundus assembly, shotgun sequence data and Chicago library sequences were used by Dovetail Genomics as input data for HiRise, as described in ref. ${ }^{22}$. Genome assembly contiguity statistics were obtained using a minimum $\mathrm{N}$ track length of 1 to delimit the contig blocks within the scaffolds.

Protein-coding gene and functional annotation. Homology-based gene prediction was performed using as a reference the Ensembl gene sets of Myotis lucifugus, Pteropus alecto, Myotis davidii, horse and human. We aligned the protein sequences of the reference gene sets to the $D$. rotundus assembly using tblastn $^{47}$ and linked the blast hits into candidate gene loci with genBlast ${ }^{48}$. We filtered out those candidate loci with homologous block length $<90 \%$ of the query length. We extracted genomic sequences of candidate gene loci, including the intronic regions and $3 \mathrm{~kb}$ upstream/downstream sequences. The sequences were passed to GeneWise ${ }^{49}$ to search for accurately spliced alignments. We filtered out pseudogenes containing more than one frame error for single-exon genes. Potentially pseudogenized single exons were removed if they were part of a multiexon gene. We then aligned protein sequences of these genes against UniProt using blastp and filtered out genes without matches. We also filtered out genes that had $>80 \%$ repeat regions. De novo gene prediction was performed with AUGUSTUS ${ }^{50}$ using a published common vampire bat transcriptome ${ }^{45}$ as a training data set and with masked transposable-element-related repeats. We filtered out partial and $<150 \mathrm{bp}$ predicted genes. Genes that aligned over $50 \%$ of their length to annotated transposable elements were filtered out. Finally, we built a non-redundant gene set with the homology-based evidence prioritized over the de novo evidence. If de novo genes were chosen in the reference gene set, we retained only those with $>30 \%$ of their length aligning against UniProt ${ }^{51}$ and that contained at least 3 exons. The integrated gene set was translated into amino-acid sequences, which were used to search the InterPro database with iprscan_4. $8^{52}$. We used BLAST to search the metabolic pathway database in $\mathrm{KEGG}^{53}$ and homologues in the SwissProt and TrEMBL databases in UniProt. The quality and annotation of the D. rotundus genome was assessed and compared to that of E. europaeus, R. ferrumequinum, M. brandtii, M. davidii, M. lucifugus and P. parnellii with BUSCO ${ }^{54}$.

Repeat annotation. Repeat annotation was performed on the genomes of D. rotundus, $P$. parnelli, $M$. lyra and $P$. vampyrus. Transposable elements were identified using RepeatMasker ${ }^{55}$ and RepeatProteinMask against the Repbase transposable element library ${ }^{56}$. We used RepeatScount, PILER-DF and RepeatModeler-1.0.5 $5^{55,57}$ to construct a de novo transposable element library, which was then used by RepeatMasker to predict repeats. We predicted tandem repeats using TRF ${ }^{58}$. LTR_Finder ${ }^{59}$ was used to detect long terminal repeats (LTRs). The Repbase-based annotations and the de novo annotations were merged.

Non-retroviral EVEs. We constructed a comprehensive library of all nonretroviral virus protein sequences available in GenBank and EMBL. We used DIAMOND ${ }^{60}$ to search these sequences against the $D$. rotundus genome. We extracted the matching amino-acid sequences and performed reciprocal blastp-like searches ${ }^{61}$ using DIAMOND with the selected subset of $D$. rotundus amino-acid sequences and the set of non-redundant protein sequences. D. rotundus genome sequences were considered of viral origin if they unambiguously matched viral 
proteins in the reciprocal best hits. Putative viral open reading frames were inferred from automated alignments, using exonerate ${ }^{62}$. For each putative D. rotundus viral peptide, we retrieved the function and predicted the taxonomic assignation by comparison to the best reciprocal blastp-like hit viral proteins. Phylogenetic analyses were performed by aligning the sequences using MAFFT $^{63}$ and curated using AliView ${ }^{64}$. Maximum-likelihood inferences were performed on each multiple amino-acid alignment using RAxML ${ }^{65}$. Support for nodes was obtained from 100 non-parametric bootstrap iterations, and the root of maximum likelihood trees was determined by midpoint rooting. We confirmed the presence of selected viruses by constructing double-indexed Illumina libraries ${ }^{66}$ on DNA derived from the spleen tissue of four different $D$. rotundus. Libraries were pooled and sequenced using 150-bp paired-end Illumina platform NextSeq 500. The raw paired-end reads were quality assessed, merged and filtered by mapping against the bacterial, human and chiropteran reference genome database with SMALT (https://www.sanger.ac.uk/resources/software/smalt/). Reads $>150$ bp were run through a viral assignment pipeline ${ }^{67}$ using blast ${ }^{68}$ to search against the viral nonredundant protein database. Viral-matching reads were assigned a taxonomy with MEGAN ${ }^{69}$. Reads matching viral sequences were manually verified by reciprocal blastx analysis. Positive viral hits were defined as those with at least two different reads matching two different proteins or two different regions within the same viral protein.

ERVs. We identified flanking LTR regions using RepeatMasker ${ }^{70}$ and RMBLAST (http://www.repeatmasker.org/RMBlast.html), Tandem Repeats Finder ${ }^{58}$ and RepBase $^{71}$ to the D. rotundus genome. We used blastn and tblastx with the retro-viral reference genome sequences in the RefSeq database of NCBI against the D. rotundus genome. Reads matching each of the RefSeq sequences were extracted and sorted by GI, collapsed by ID and manually verified by reciprocal blastn. Tblastx was re-run and the kept reads were mapped to the D. rotundus genome with SMALT and manually verified by reciprocal blastp. ERV sequences validation was carried out with tblastx against the Retroviridae. The retrieved ERV sequences were mapped using SMALT v0.7.6 against the genomes of D. rotundus, Mastomys natalensis, Eptesicus fuscus, $M$. lucifugus, M. brandtii, $M$. davidii, P. alecto, R. aegyptiacus and P. parnellii. Tblastx of the full genomic sequences of DrERV and DrgERV was also performed against Chiroptera. Selected genomic contigs were further analysed by reciprocal blastx against the non-redundant GenBank coding DNA sequence (CDS) translations + PDB + SwissProt + PIR + PRF database restricting the search to the Retroviridae, or against the DrgERV sequences. Retrieved sequences were aligned using MUSCLE in SeaView ${ }^{72}$. The best-fit amino-acid substitution model for each alignment was identified using jModelTest $2^{73}$ and trees were inferred under maximum-likelihood criteria using RAxML ${ }^{65}$.

Orthologous gene families. Using the $D$. rotundus genome against a range of other mammalian species, we performed clustering of orthologous genes using two strategies. (1) Identifying single-copy orthologues in the species by using the TreeFam method $^{74}$. (2) Identifying 1:1 orthologues by building pair-wise orthologues between $D$. rotundus and the other species and using a reciprocal best hit $(\mathrm{RBH})$ plus synteny approach as described in ref. ${ }^{75}$.

dN/dS analyses. We used PAML codeml ${ }^{76}$ on the cleaned CDS alignments from the two sets of orthologous families and the corresponding phylogenetic tree as reported in ref. ${ }^{77}$. To identify genes with accelerated evolution in the D. rotundus lineage we ran the two-ratio branch model. As a null model, we used the one-ratio model. Using these results, we performed likelihood ratio tests (LRTs) to identify genes with significant $P$ values. To adjust for multiple testing, we used the FDR method. To identify genes with positively selected sites in D. rotundus, we also used a branch site with PAML codeml ${ }^{76}$. LRT and FDR were computed as carried out for the branch model tests.

Gene family expansion/contraction. We used $\mathrm{CAFE}^{78}$ with the results from the single-copy orthologous gene families. To obtain the dated tree required as input for CAFE, we obtained divergence times from the fossil dating records from TimeTree ${ }^{79}$. We concatenated the CDSs aligned with MAFFT and used PAML mcmctree to determine split times with the approximate likelihood calculation method. Genes with >200 copies in 1 of the species were filtered out.

Putative gene loss. We identified genes putatively lost in D. rotundus as previously described in ref. ${ }^{80}$. The human gene set was downloaded from Ensembl and we obtained a non-redundant gene catalogue to be used as a reference by collapsing redundant genes and keeping the longest open reading frame. We used Bos taurus, Equus caballus and E. europaeus as outgroups. Genes identified as missing in $D$. rotundus were searched with blastp against the protein catalogues of the other available bats. Further validation on selected genes was performed by: evaluating the conservation of their syntenic regions compared to other species, and the GC content of the syntenic regions compared to the D. rotundus genome average GC content; searching them against the published $D$. rotundus transcriptome; and through PCR assays.
Functional characterization. We performed GO analysis using GOrilla ${ }^{81}$ as well as manual characterization using the GO annotations of the human genes downloaded from Ensembl and literature mining. We used PROVEAN ${ }^{82}$ to characterize the functional impact of the non-synonymous substitutions present only on the proteins in $D$. rotundus.

Species-specific PSS identification and protein modelling. We tested for positive selection and positive selected sites (PSSs) using the complete CDS alignments for the proteins FFAR1, PLXNA4, REG4, RNAS7 and TA2R3 from various species downloaded from the OrthoMaM database ${ }^{83}$. Sequences were realigned using MUSCLE $^{84}$ in SeaView ${ }^{72}$ and phylogenetic analysis was performed using PhyML ${ }^{85}$. We used PAML codeml ${ }^{76}$ to test: the M1/M2 branch model constraining the D. rotundus node ${ }^{76}$; the M8a/M8 site model; and the branch-site model A constraining the $D$. rotundus node. The branch-site model A was evaluated under a LRT against the null hypothesis, while PSSs were scored under naïve empirical Bayes (NEB) and Bayes empirical Bayes (BEB). We performed modelling of the mentioned proteins from D. rotundus using the PyMOL Molecular Graphics System (Schrödinger, LLC). The three-dimensional protein models of the D. rotundus sequences were constructed using Phyre $2^{86}$ based on profile hidden Markov model analysis using the structures reported in refs ${ }^{87-90}$.

Metagenomic data. We used faecal and anal swab samples from $D$. rotundus, $R$. aegyptiacus, $R$. ferrumequinum and $M$. gigas. The D. rotundus samples were obtained from wild $D$. rotundus individuals captured using mist nets and cloth bags and preserved in RNAlater according to the manufacturer's instructions. The $R$. ferrumequinum samples were collected in Woodchester Mansion, Gloucestershire, UK. The M. gigas samples from Australia were collected by the consulting company Biologic in Pilbarra, Australia. The R. aegyptiacus faecal samples were collected from the Copenhagen Zoo. We have complied with all relevant ethical regulations for the collection of these samples. DNA from faecal samples was extracted using the PowerFecal DNA Isolation Kit (MoBio) with modifications to the manufacturer's instructions. DNA from the D. rotundus milk sample was extracted using a standard commercial kit (Qiagen), following the manufacturer's instructions. Anal swab samples were extracted using the BiOstic Bacteremia DNA Isolation Kit (MoBio) following the manufacturer's protocol. All samples were kept at $-20^{\circ} \mathrm{C}$. Libraries were built using the NEBnext DNA Library Prep Mast Mix Set for 454 (New England BioLabs) following the manufacturer's instructions. Samples were 100-bp paired-end sequenced on the Illumina 2500 HiSeq platform.

Taxonomic and functional identification. The reads were cleaned with Trimmomatic ${ }^{11}$ and prinseq-lite ${ }^{92}$. The data sets were mapped against the closest available bat genome and only the non-mapping reads were kept. MGmapper ${ }^{93}$ was used for the taxonomic identification of invertebrates, protozoa, fungi, virus and bacteria. We kept the species identified with a coverage value higher than the firstquartile from the coverage distribution of the corresponding database and filtered out those found on the corresponding extraction blanks. Rarefaction curves from each data set were obtained using an in-house script with the MGmapper results. We then performed de novo assembly using IDBA_UD ${ }^{94}$, predicted genes using Prodigal ${ }^{95}$ and generated a non-redundant gene catalogue with usearch ${ }^{96}$. The non-redundant catalogue was searched with ublast ${ }^{96}$ against the UniProt database $\mathrm{e}^{51}$ for functional and taxonomic annotation. We used DIAMOND ${ }^{60}$ to search the unmapped reads against the UniProt database keeping only the best hit for functional and taxonomic annotation. We annotated the UniProt protein identifications using KEGG orthology (KO) and eggNOG IDs.

Taxonomic and functional metagenomics comparison. We filtered on the basis of the breadth of coverage and the identifications of the extraction blanks. We removed any non-microbial hit and any taxa in which the paired reads matched different genera or only one of the reads had a hit. The counts were normalized by percentage. We identified a microbial taxonomic and functional sanguivorous core by comparing the filtered sets of the bats and keeping as core those taxa and genes identified only in the $D$. rotundus samples. We manually examined the taxa from the filtered taxonomic identifications, and the KO and COG annotations from the filtered non-redundant gene set catalogue. We compared the normalized abundance of taxa and functions between $D$. rotundus and the non-sanguivorous bats as follows. (1) Using the distributions of the different functional categories from $D$. rotundus and each non-sanguivorous bat species with a Wilcoxon ranksum test. (2) Using the entire taxonomic and functional data sets, as well as down-sampling the normalized count values. Sampling values were the minimum, median and third-quartile values of the count distributions. With the resulting data sets, we calculated the Euclidean, Bray-Curtis and Jaccard distance metrics with the $\mathrm{R}$ package vegan ${ }^{97}$, and used the Ward hierarchical clustering method using UPGMA and Ward, and performed PCAs with prcomp and the GPA method. (3) We identified taxa and functions significantly contributing to the variation between the $D$. rotundus and the non-sanguivorous bat species. We examined the rotation matrix from the PCA of the normalized counts, excluding the four deepest sequenced samples, of the species and genus microbial taxonomic levels and the KEGG functional pathways. We identified the most significantly abundant 
D. rotundus microbial taxa as those with a significantly higher median normalized count value $(P<0.05)$ in $D$. rotundus and a median and mean normalized count value of 0 in the other 3 bat species for the first 3 principal components. We also identified significantly more abundant genes in D. rotundus by generating and annotating with KEGG a non-redundant gene set with all of the predicted genes from all of the bat samples. The reads of the samples were mapped against this bat non-redundant gene set, and a normalized count matrix was generated and used for Fisher tests on each of the functional pathways.

Life Sciences Reporting Summary. Further information on experimental design is available in the Life Sciences Reporting Summary.

Code availability. In-house scripts used for the processing of the data are available from the corresponding authors upon request.

Data availability. The NCBI BioProject accession code for the genome assembly is PRJNA414273, and the sequence reads are available at the NCBI sequence read archive (SRA) under the accession SRA619672. The BioProject code for the metagenomic sequencing data is PRJNA415003, and the reads can be accessed at SRA with the accession SRA620977.

Received: 23 August 2016; Accepted: 11 January 2018; Published online: 19 February 2018

\section{References}

1. Breidenstein, C. P. Digestion and assimilation of bovine blood by a vampire bat (Desmodus rotundus). J. Mammal. 63, 482-484 (1982).

2. Edwards, M. A., Kaufman, M. L. \& Storvick, C. A. Microbiologic assay for the thiamine content of blood of various species of animals and man. Am. J. Clin. Nutr. 5, 51-55 (1957).

3. Gracheva, E. O. et al. Molecular basis of infrared detection by snakes. Nature 464, 1006-1011 (2010).

4. Kishida, R., Goris, R. C., Terashima, S. \& Dubbeldam, J. L. A suspected infrared-recipient nucleus in the brainstem of the vampire bat, Desmodus rotundus. Brain. Res. 322, 351-355 (1984).

5. Singer, M. A. Vampire bat, shrew, and bear: comparative physiology and chronic renal failure. Am. J. Physiol. Regul. Integr. Comp. Physiol. 282, R1583-R1592 (2002)

6. Escalera-Zamudio, M. et al. The evolution of bat nucleic acid sensing Toll-like receptors. Mol. Ecol. 24, 5899-5909 (2015).

7. Ley, R. E., Lozupone, C. A., Hamady, M., Knight, R. \& Gordon, J. I. Worlds within worlds: evolution of the vertebrate gut microbiota. Nat. Rev. Microbiol. 6, 776-788 (2008).

8. Graf, J., Kikuchi, Y. \& Rio, R. V. M. Leeches and their microbiota: naturally simple symbiosis models. Trends Microbiol. 14, 365-371 (2006).

9. Hornborstel, H. Ueber die bakteriologischen Eigenschaften des Darmsymbionten beim medizinischen Blutegel (Hirudo officinalis) nebst Bemerkungen zur Symbiosefrage. Zbl. Bakteriol. 148, 36-47 (1942)

10. Graf, J. The effect of symbionts on the physiology of Hirudo medicinalis, the medicinal leech. Invertebr. Reprod. Dev. 41, 269-275 (2002).

11. Indergand, S. \& Graf, J. Ingested blood contributes to the specificity of the symbiosis of Aeromonas veronii Biovar Sobria and Hirudo medicinalis, the medicinal leech. Appl. Environ. Microbiol. 66, 4735-4741 (2000).

12. Semova, I. et al. Microbiota regulate intestinal absorption and metabolism of fatty acids in the zebrafish. Cell Host Microbe 12, 277-288 (2012).

13. Hajela, N. et al. Gut microbiome, gut function, and probiotics: implications for health. Indian I. Gastroenterol. 34, 93-107 (2015).

14. Cryan, J. F. \& Dinan, T. G. Mind-altering microorganisms: the impact of the gut microbiota on brain and behaviour. Nat. Rev. Neurosci. 13, 701-712 (2012).

15. Qin, J. et al. A metagenome-wide association study of gut microbiota in type 2 diabetes. Nature 490, 55-60 (2012)

16. Ley, R. E., Turnbaugh, P. J., Klein, S. \& Gordon, J. I. Microbial ecology: human gut microbes associated with obesity. Nature 444, 1022-1023 (2006).

17. Sokol, H. et al. Faecalibacterium prausnitzii is an anti-inflammatory commensal bacterium identified by gut microbiota analysis of Crohn disease patients. Proc. Natl Acad. Sci. USA 105, 16731-16736 (2008).

18. Jefferson, R. The Hologenome. Agriculture, Environment and the Developing World: A Future of PCR. VHS Recording, Cold Spring Harbor Laboratory Press, Part 4: The Hologenome Plenary Lecture (Cold Spring Harbor Laboratory Press, New York, 1994).

19. Bordenstein, S. R. \& Theis, K. R. Host biology in light of the microbiome: ten principles of holobionts and hologenomes. PLoS Biol. 13, e1002226 (2015).

20. Ballal, S. A., Gallini, C. A., Segata, N., Huttenhower, C. \& Garrett, W. S. Host and gut microbiota symbiotic factors: lessons from inflammatory bowel disease and successful symbionts. Cell. Microbiol. 13, 508-517 (2011).

21. Smith, J. D. L. \& Gregory, T. R. The genome sizes of megabats (Chiroptera: Pteropodidae) are remarkably constrained. Biol. Lett. 5, 347-351 (2009).
22. Putnam, N. H. et al. Chromosome-scale shotgun assembly using an in vitro method for long-range linkage. Genome Res. 26, 342-350 (2015).

23. Kojima, K. K. \& Jurka, J. Crypton transposons: identification of new diverse families and ancient domestication events. Mob. DNA 2, 12 (2011).

24. Robertson, H. M. Members of the pogo superfamily of DNA-mediated transposons in the human genome. Mol. Gen. Genet. 252, 761-766 (1996).

25. Aiewsakun, P. \& Katzourakis, A. Endogenous viruses: Connecting recent and ancient viral evolution. Virology 479-480, 26-37 (2015).

26. Hayward, J. A. et al. Identification of diverse full-length endogenous betaretroviruses in megabats and microbats. Retrovirology 10, 35 (2013).

27. Escalera-Zamudio, M. et al. A novel endogenous betaretrovirus in the common vampire bat (Desmodus rotundus) suggests multiple independent infection and cross-species transmission events. J. Virol. 89, 5180-5184 (2015)

28. Hong, W. \& Zhao, H. Vampire bats exhibit evolutionary reduction of bitter taste receptor genes common to other bats. Proc. R. Soc. B 281, 20141079 (2014)

29. Dotson, C. D. et al. Bitter taste receptors influence glucose homeostasis. PLoS ONE 3, e3974 (2008)

30. Phillips, C. D. \& Baker, R. J. Secretory gene recruitments in vampire bat salivary adaptation and potential convergences with sanguivorous leeches. Front. Ecol. Evol. 3, 122 (2015).

31. Gracheva, E. O. et al. Ganglion-specific splicing of TRPV1 underlies infrared sensation in vampire bats. Nature 476, 88-91 (2011)

32. Wang, Y. The functional regulation of TRPV1 and its role in pain sensitization. Neurochem. Res. 33, 2008-2012 (2008).

33. Phillips, C. D. et al. Microbiome analysis among bats describes influences of host phylogeny, life history, physiology and geography. Mol. Ecol. 21 2617-2627 (2012)

34. Apitz-Castro, R. et al. Purification and partial characterization of draculin, the anticoagulant factor present in the saliva of vampire bats (Desmodus rotundus). Thromb. Haemost. 73, 94-100 (1995).

35. Truelove, E., Fielding, A. K. \& Hunt, B. J. The coagulopathy and thrombotic risk associated with L-asparaginase treatment in adults with acute lymphoblastic leukaemia. Leukemia 27, 553-559 (2013).

36. Kikuchi, Y. \& Graf, J. Spatial and temporal population dynamics of a naturally occurring two-species microbial community inside the digestive tract of the medicinal leech. Appl. Environ. Microbiol. 73, 1984-1991 (2007).

37. Worthen, P. L., Gode, C. J. \& Graf, J. Culture-independent characterization of the digestive-tract microbiota of the medicinal leech reveals a tripartite symbiosis. Appl. Environ. Microbiol. 72, 4775-4781 (2006).

38. Guyton, A. C., Coleman, T. G., Young, D. B., Lohmeier, T. E. \& DeClue, J. W. Salt balance and long-term blood pressure control. Annu. Rev. Med. 31, 15-27 (1980).

39. Bar-Peled, L., Schweitzer, L. D., Zoncu, R. \& Sabatini, D. M. Ragulator is a GEF for the Rag GTPases that signal amino acid levels to mTORC1. Cell 150, 1196-1208 (2012).

40. Donohoe, D. R. et al. The microbiome and butyrate regulate energy metabolism and autophagy in the mammalian colon. Cell. Metab. 13, 517-526 (2011)

41. Huq, A. H., Lovell, R. S., Ou, C. N., Beaudet, A. L. \& Craigen, W. J. X-linked glycerol kinase deficiency in the mouse leads to growth retardation, altered fat metabolism, autonomous glucocorticoid secretion and neonatal death. Hum. Mol. Genet. 6, 1803-1809 (1997).

42. August, P. R. et al. Biosynthesis of the ansamycin antibiotic rifamycin: deductions from the molecular analysis of the rif biosynthetic gene cluster of Amycolatopsis mediterranei S699. Chem. Biol. 5, 69-79 (1998).

43. Luo, R. et al. SOAPdenovo2: an empirically improved memory-efficient short-read de novo assembler. Gigascience 1, 18 (2012).

44. Liu, B. et al. Estimation of genomic characteristics by analyzing k-mer frequency in de novo genome projects. Preprint at https://arxiv.org/ abs/1308.2012 (2013).

45. Francischetti, I. M. B. et al. The 'Vampirome': transcriptome and proteome analysis of the principal and accessory submaxillary glands of the vampire bat Desmodus rotundus, a vector of human rabies. J. Proteom. 82 288-319 (2013).

46. Kent, W. J. BLAT-the BLAST-like alignment tool. Genome Res. 12, 656-664 (2002).

47. Altschul, S. F., Gish, W., Miller, W., Myers, E. W. \& Lipman, D. J. Basic local alignment search tool. J. Mol. Biol. 215, 403-410 (1990).

48. She, R., Chu, J. S.-C., Wang, K., Pei, J. \& Chen, N. GenBlastA: enabling BLAST to identify homologous gene sequences. Genome Res. 19, 143-149 (2009).

49. Birney, E., Clamp, M. \& Durbin, R. GeneWise and Genomewise. Genome Res 14, 988-995 (2004)

50. Stanke, M. et al. AUGUSTUS: ab initio prediction of alternative transcripts. Nucleic Acids Res. 34, W435-W439 (2006).

51. UniProt Consortium. The universal protein resource (UniProt). Nucleic Acids Res. 36, D190-D195 (2008). 
52. Hunter, S. et al. InterPro: the integrative protein signature database. Nucleic Acids Res. 37, D211-D215 (2009).

53. Kanehisa, M. \& Goto, S. KEGG: Kyoto encyclopedia of genes and genomes. Nucleic Acids Res. 28, 27-30 (2000).

54. Simão, F. A., Waterhouse, R. M., Ioannidis, P., Kriventseva, E. V. \& Zdobnov, E. M. BUSCO: assessing genome assembly and annotation completeness with single-copy orthologs. Bioinformatics 31, 3210-3212 (2015)

55. Smit, A., Hubley, R. \& Green, P. RepeatMasker Open-3.0; http://www. repeatmasker.org.

56. Jurka, J. Repeats in genomic DNA: mining and meaning. Curr. Opin. Struct. Biol. 8, 333-337 (1998)

57. Smit, A. \& Hubley, R. RepeatModeler Open-1.0; http://www.repeatmasker. org/RepeatModeler.

58. Benson, G. Tandem repeats finder: a program to analyze DNA sequences. Nucleic Acids Res. 27, 573-580 (1999).

59. Xu, Z. \& Wang, H. LTR_FINDER: an efficient tool for the prediction of full-length LTR retrotransposons. Nucleic Acids Res. 35, W265-W268 (2007).

60. Buchfink, B., Xie, C. \& Huson, D. H. Fast and sensitive protein alignment using DIAMOND. Nat. Methods 12, 59-60 (2014).

61. Altschul, S. F. et al. Gapped BLAST and PSI-BLAST: a new generation of protein database search programs. Nucleic Acids Res. 25, 3389-3402 (1997).

62. Slater, G. S. C. \& Birney, E. Automated generation of heuristics for biological sequence comparison. BMC Bioinformatics 6, 31 (2005).

63. Katoh, K. \& Standley, D. M. MAFFT multiple sequence alignment software version 7: improvements in performance and usability. Mol. Biol. Evol. 30, 772-780 (2013).

64. Larsson, A. AliView: a fast and lightweight alignment viewer and editor for large datasets. Bioinformatics 30, 3276-3278 (2014).

65. Stamatakis, A. RAxML-VI-HPC: maximum likelihood-based phylogenetic analyses with thousands of taxa and mixed models. Bioinformatics 22, 2688-2690 (2006).

66. Meyer, M. \& Kircher, M. Illumina sequencing library preparation for highly multiplexed target capture and sequencing. Cold Spring Harb. Protoc. 2010, pdb.prot5448 (2010).

67. Taboada, B. et al. Is there still room for novel viral pathogens in pediatric respiratory tract infections?. PLoS ONE 9, e113570 (2014).

68. Camacho, C. et al. BLAST+: architecture and applications. BMC Bioinformatics 10, 421 (2009).

69. Huson, D. H. \& Weber, N. Microbial community analysis using MEGAN. Methods Enzymol. 531, 465-485 (2013).

70. Tarailo-Graovac, M. \& Chen, N. UNIT 4.10 Using RepeatMasker to identify repetitive elements in genomic sequences. Curr. Protoc. Bioinformatics $\mathbf{2 5}$ 4.10.1-4.10.14 (2009).

71. Jurka, J. Repbase update: a database and an electronic journal of repetitive elements. Trends Genet. 16, 418-420 (2000).

72. Gouy, M., Guindon, S. \& Gascuel, O. SeaView version 4: a multiplatform graphical user interface for sequence alignment and phylogenetic tree building. Mol. Biol. Evol. 27, 221-224 (2010).

73. Darriba, D., Taboada, G. L., Doallo, R. \& Posada, D. jModelTest 2: more models, new heuristics and parallel computing. Nat. Methods 9, 772 (2012).

74. Schreiber, F., Patricio, M., Muffato, M., Pignatelli, M. \& Bateman, A. TreeFamv9: a new website, more species and orthology-on-the-fly. Nucleic Acids Res. 42, D922-D925 (2014).

75. Jarvis, E. D. et al. Whole genome analyses resolve early branches in the tree of life of modern birds. Science 346, 1320-1331 (2014).

76. Yang, Z. PAML 4: phylogenetic analysis by maximum likelihood. Mol. Biol. Evol. 24, 1586-1591 (2007).

77. Teeling, E. C. et al. A molecular phylogeny for bats illuminates biogeography and the fossil record. Science 307, 580-584 (2005).

78. De Bie, T., Cristianini, N., Demuth, J. P. \& Hahn, M. W. CAFE: a computational tool for the study of gene family evolution. Bioinformatics $\mathbf{2 2}$ 1269-1271 (2006).

79. Hedges, S. B., Marin, J., Suleski, M., Paymer, M. \& Kumar, S. Tree of life reveals clock-like speciation and diversification. Mol. Biol. Evol. 32, 835-845 (2015).

80. Zhang, G. et al. Comparative genomics reveals insights into avian genome evolution and adaptation. Science 346, 1311-1320 (2014).

81. Eden, E., Navon, R., Steinfeld, I., Lipson, D. \& Yakhini, Z. GOrilla: a tool for discovery and visualization of enriched GO terms in ranked gene lists. BMC Bioinformatics 10, 48 (2009).

82. Choi, Y. \& Chan, P. A. PROVEAN web server: a tool to predict the functiona effect of amino acid substitutions and indels. Bioinformatics 31, 2745-2747 (2015).

83. Ranwez, V. et al. OrthoMaM: a database of orthologous genomic markers for placental mammal phylogenetics. BMC Evol. Biol. 7, 241 (2007).

84. Edgar, R. C. MUSCLE: multiple sequence alignment with high accuracy and high throughput. Nucleic Acids Res. 32, 1792-1797 (2004).

85. Guindon, S. et al. New algorithms and methods to estimate maximumlikelihood phylogenies: assessing the performance of PhyML 3.0. Syst. Biol. 59, 307-321 (2010).
86. Kelley, L. A., Mezulis, S., Yates, C. M., Wass, M. N. \& Sternberg, M. J. E. The Phyre 2 web portal for protein modeling, prediction and analysis. Nat. Protoc. 10, 845-858 (2015).

87. Kang, Y. et al. Crystal structure of rhodopsin bound to arrestin by femtosecond X-ray laser. Nature 523, 561-567 (2015)

88. He, H., Yang, T., Terman, J. R. \& Zhang, X. Crystal structure of the plexin A3 intracellular region reveals an autoinhibited conformation through active site sequestration. Proc. Natl Acad. Sci. USA 106, 15610-15615 (2009).

89. Ho, M.-R. et al. Human RegIV protein adopts a typical C-type lectin fold but binds Mannan with two calcium-independent sites. J. Mol. Biol. 402, 682-695 (2010).

90. Huang, Y.-C. et al. The flexible and clustered lysine residues of human ribonuclease 7 are critical for membrane permeability and antimicrobial activity. J. Biol. Chem. 282, 4626-4633 (2007).

91. Bolger, A. M., Lohse, M. \& Usadel, B. Trimmomatic: a flexible trimmer for Illumina sequence data. Bioinformatics 30, 2114-2120 (2014).

92. Schmieder, R. \& Edwards, R. Quality control and preprocessing of metagenomic datasets. Bioinformatics 27, 863-864 (2011).

93. Hasman, H. et al. Rapid whole-genome sequencing for detection and characterization of microorganisms directly from clinical samples. J. Clin. Microbiol. 52, 3136-3136 (2014).

94. Peng, Y., Leung, H. C. M., Yiu, S. M. \& Chin, F. Y. L. IDBA-UD: a de novo assembler for single-cell and metagenomic sequencing data with highly uneven depth. Bioinformatics 28, 1420-1428 (2012).

95. Hyatt, D. et al. Prodigal: prokaryotic gene recognition and translation initiation site identification. BMC Bioinformatics 11, 119 (2010).

96. Edgar, R. C. Search and clustering orders of magnitude faster than BLAST. Bioinformatics 26, 2460-2461 (2010).

97. Oksanen, J. et al. vegan: Community Ecology Package. R package version 2.3-5. (R Foundation for Statistical Computing, Vienna, 2016).

\section{Acknowledgements}

We thank M. L. Méndez Ojeda, G. Czirjak, L. Caballero, O. Ríos, A. Patraca, C. Tello, D. Becker and M.E. Roelke-Parker for the collection and exportation of vampire bat samples, as well as P. Perelman and M. Korody for the preparation of genomic DNA. We thank Dovetail Genomics for the improvement of the genome contiguity of D. rotundus, in particular R. E. Green, M. Hartley and B. Rice. We also thank G. Jones for his support in the capture of the R. ferrumequinum bat samples and for useful comments on the manuscript, and R. Dunn and L. Nichols for their support at the early stages of the project. Likewise, we thank A. Alberdi and O. Aizpurua for assistance regarding samples from Rhinolophus euryale, Miniopterus schreibersii, Nyctalus leisleri and Nyctalus lasiopterus. We thank the Danish National High-Throughput DNA Sequencing Centre for assistance in generating the metagenome and Dovetail Illumina sequence data. We also thank the Instituto de Biotecnologia-UNAM for giving us access to its computer cluster and J. Verleyenfor his computer support. M.T.P.G. and M.L.Z.M. recognize Lundbeck Foundation R52-A5062, ERC Consolidator Grant (681396 Extinction Genomics) and Danish National Research Foundation DNRF94 grants as the principal funding behind this work. M.E.-Z. and A.D.G. acknowledge the Deutsche Forschungsgemeinschaft (DFG) (GR 3924/9-1 to A.D.G) and an international doctoral scholarship provided by the Consejo Nacional de Ciencia y Tecnología (CONACyT) and the German Academic Exchange Service (DAAD) (311664). O.G.P. and J.T. acknowledge funding from the European Research Council under FP7/2007-2013/ERC grant agreement no. 614725-PATHPHYLODYN. A.K. recognizes funding from the Royal Society. H.K.F. acknowledges funding from a Bing-Mooney Fellowship in Environmental Science and Conservation. Field work in Peru was funded by the US National Science Foundation (grant DEB-1020966) and a Sir Henry Dale Fellowship to D.S., jointly funded by the Wellcome Trust and the Royal Society (grant 102507/Z/13/Z). Funding for Costa Rican fieldwork came from the Stanford Woods Institute for the Environment Environmental Venture Project.

\section{Author contributions}

M.L.Z.M. and Z.X. contributed equally to the work. M.L.Z.M. and M.T.P.G. devised the study and supervised all parts of the project. M.L.Z.M., Z.X., M.E.-Z., S.L. and G.Z designed the genomic and comparative genomic methods. M.L.Z.M. and T.S.-P. designed the metagenomics methods. M.L.Z.M. led the data integration, bioinformatic analyses and carried out the primary interpretation of analytical outcomes in close collaboration with M.E.-Z., A.D.G., D.S and M.T.P.G. The design of the viral analyses was carried out by J.T., A.K., O.G.P., M.E.-Z., U.L. and B.T., as was the performance of the viral bioinformatic analyses. Z.X., Y.L., S.L. and M.L.Z.M. performed the genomic methods. A.C.B. and J.A.S.C. provided support on a variety of bioinformatic analyses. S.L. performed the laboratory protocols for the genomic analysis. G.P. performed validation of the chosen putatively lost genes. O.A.R., M.B., T.S.-P., M.T.P.G. and G.Z. aided in the generation of the common vampire bat genome. A.K.R. and M.E.-Z. performed the laboratory metagenomic protocols. H.K.F., E.L.-R., E.R.-A., K.B., M.F.B. and N.E.W. carried out the collection and export of samples for the metagenomic data sets. C.F.A., E.R.-A. and E.L.-R. carried out the collection of the samples used for the viral analyses. M.L.Z.M. drafted the manuscript. M.E.-Z., J.T., D.S., H.K.F., K.B., A.D.G., M.F.B., M.B., G.Z., T.S.-P. and M.T.P.G. provided critical and substantial contribution to the various draft versions of the paper. All authors approved the final version. 


\section{Competing interests}

The authors declare no competing financial interests.

\section{Additional information}

Supplementary information is available for this paper at https://doi.org/10.1038/ s41559-018-0476-8.

Reprints and permissions information is available at www.nature.com/reprints. Correspondence and requests for materials should be addressed to M.L.Z. or T.S. or M.P.T.G.

Publisher's note: Springer Nature remains neutral with regard to jurisdictional claims in published maps and institutional affiliations.

(c) (1)

Open Access This article is licensed under a Creative Commons Attribution 4.0 International License, which permits use, sharing, adaptation, distribution and reproduction in any medium or format, as long as you give appropriate credit to the original author(s) and the source, provide a link to the Creative Commons license, and indicate if changes were made. The images or other third party material in this article are included in the article's Creative Commons license, unless indicated otherwise in a credit line to the material. If material is not included in the article's Creative Commons license and your intended use is not permitted by statutory regulation or exceeds the permitted use, you will need to obtain permission directly from the copyright holder. To view a copy of this license, visit http://creativecommons.org/licenses/by/4.0/. 


\section{natureresearch}

Corresponding author(s): Marie Lisandra Zepeda Mendoza

Initial submission

Revised version

$\checkmark$ Final submission

\section{Life Sciences Reporting Summary}

Nature Research wishes to improve the reproducibility of the work that we publish. This form is intended for publication with all accepted life science papers and provides structure for consistency and transparency in reporting. Every life science submission will use this form; some list items might not apply to an individual manuscript, but all fields must be completed for clarity.

For further information on the points included in this form, see Reporting Life Sciences Research. For further information on Nature Research policies, including our data availability policy, see Authors \& Referees and the Editorial Policy Checklist.

\section{- Experimental design}

1. Sample size

Describe how sample size was determined.

We obtained as many samples as possible form the chosen species to perform our metagenomics comparisons.

2. Data exclusions

Describe any data exclusions.

\section{Replication}

Describe whether the experimental findings were reliably reproduced.

We used all the generated data. Different sets for the different analyses, each one described in the extended methods. For example, the identification of the microbiome cores was done using all the datasets, and the microbiome profiles distances were obtained both using all the sets downsampled, and excluding the 3 most heavily sequenced samples to avoid bias

4. Randomization

Describe how samples/organisms/participants were allocated into experimental groups.

\section{All attempts at replication were successful}

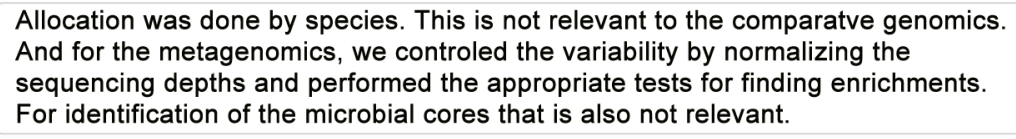

5. Blinding

Describe whether the investigators were blinded to group allocation during data collection and/or analysis.

Blinding is not relevant to our analyses, which compare the genomes and metagenomes of the species, our analyses do not make predictions of their microbes/genes, but characterize their identifications.

Note: all studies involving animals and/or human research participants must disclose whether blinding and randomization were used.

6. Statistical parameters

For all figures and tables that use statistical methods, confirm that the following items are present in relevant figure legends (or in the

Methods section if additional space is needed).

n/a $\mid$ Confirmed

X The exact sample size $(n)$ for each experimental group/condition, given as a discrete number and unit of measurement (animals, litters, cultures, etc.)

A description of how samples were collected, noting whether measurements were taken from distinct samples or whether the same

X sample was measured repeatedly

X A statement indicating how many times each experiment was replicated

The statistical test(s) used and whether they are one- or two-sided (note: only common tests should be described solely by name; more

Complex techniques should be described in the Methods section)

X A description of any assumptions or corrections, such as an adjustment for multiple comparisons

$\mathrm{X}$ The test results (e.g. $P$ values) given as exact values whenever possible and with confidence intervals noted

$\mathrm{X}$ A clear description of statistics including central tendency (e.g. median, mean) and variation (e.g. standard deviation, interquartile range)

$\mathrm{X} \square$ Clearly defined error bars 
Policy information about availability of computer code

\section{Software}

Describe the software used to analyze the data in this study.

We used many different softwares. All of them are specified in the Methods sections in the main and supplementary text, including the versions. In house scripts upon request.

For manuscripts utilizing custom algorithms or software that are central to the paper but not yet described in the published literature, software must be made available to editors and reviewers upon request. We strongly encourage code deposition in a community repository (e.g. GitHub). Nature Methods guidance for providing algorithms and software for publication provides further information on this topic.

\section{- Materials and reagents}

Policy information about availability of materials

8. Materials availability

Indicate whether there are restrictions on availability of unique materials or if these materials are only available for distribution by a for-profit company.

9. Antibodies

Describe the antibodies used and how they were validated for use in the system under study (i.e. assay and species).

\section{Eukaryotic cell lines}

a. State the source of each eukaryotic cell line used.

b. Describe the method of cell line authentication used.

c. Report whether the cell lines were tested for mycoplasma contamination.

d. If any of the cell lines used are listed in the database of commonly misidentified cell lines maintained by ICLAC, provide a scientific rationale for their use.
Laboratory reagents are all comercially available. The unique materials are the bat fecal samples and Desmodus rotundus skin cells, which were all used for the generation of the sequencing data.

We did not use any antibodies

We did not use any cell line. We generated the $D$. rotundus genome with cultured cells from the San Diego Zoo collection, that were originally derived from a skin sample, as mentioned in the methods section

No eukaryotic cell lines were used

No eukaryotic cell lines were used

N/A

\section{- Animals and human research participants}

Policy information about studies involving animals; when reporting animal research, follow the ARRIVE guidelines

11. Description of research animals

Provide details on animals and/or animal-derived materials used in the study.
We used samples from Desmodus rotundus, Rousettus aegyptiacus, Rhinolophus ferrumequinum, Macroderma gigas, Rhinolophus euryale, Miniopterus schreibersii, Nyctalus leisleri, and Nyctalus lasiopterus. Physiological information of the vampire individuals sampled by net capturing is presented in the Supplementary Information.

\section{Policy information about studies involving human research participants}

12. Description of human research participants

Describe the covariate-relevant population characteristics of the human research participants.
The study did not involve human research participants 